The following review was printed in our sister publication, The Journal of Clinical Psychiatry. We at the Companion believe that the review will be of interest to our readers as well. - L.C.

\section{Bipolar Disorder:}

A Guide for Patients and Families, 2nd ed.

by Francis Mark Mondimore, M.D. Johns Hopkins

University Press, Baltimore, Md., 2006, in Johns Hopkins

Press Health Book series, 304 pages, $\$ 45.00$ (hardcover), $\$ 18.95$ (paper).

\section{Depression: The Mood Disease, 3rd ed.}

by Francis Mark Mondimore, M.D. Johns Hopkins University Press, Baltimore, Md., 2006, in Johns Hopkins Press Health Book series, 224 pages, $\$ 45.00$ (hardcover), $\$ 19.95$ (paper).

These 2 delightful volumes are written for patients and relatives of patients, although their information also would make them of interest to medical students and psychiatric residents. There is considerable overlap in topics-both have discussions of the brain and neurotransmitters, a review of medications used to treat depression, a review of psychotherapies used to treat these disorders, and a discussion of other treatments including ECT, transcranial magnetic stimulation, and vagus nerve stimulation. Both volumes include up-to-date information on newer antidepressants as well as other psychopharmacologic treatments, such as neuroleptics and mood stabilizers. Also, each volume has a description of special circumstances (disorders among children, adolescents, elderly, women, etc.).

Both volumes are liberally complemented with case studies to illustrate particular points-for example, case studies that describe different types of presentations or comorbid conditions. The bipolar volume also has chapters on genetics and seasonal mood disorders, and both volumes list resources for patients such as the Depression Bipolar Support Alliance, and e-mail as well as street addresses and phone numbers are included.

Unlike many volumes written for patients and families, each volume is well indexed and the bipolar (but not the major depression) volume has a list of pertinent journal references for each chapter. There are sufficient graphics and tables for the basic mechanisms of the disorders as well as the agents used in treatment.

In an era of underdiagnosis and undertreatment of both major depression and bipolar disorders, anything that would increase public awareness of these conditions is helpful. Such volumes, to be useful, should contain accurate information, be current, and be comprehensive without presenting too much information. Dr. Mondimore has succeeded on most of these points, although at times I thought the volumes might be a bit too technical for many patients and their families. However, given the choice of being too technical versus less inclusive, having more information is probably better-if patients do not fully understand what is presented, they can discuss these areas further with their clinician.
These are practical volumes for patients and their relatives, and I will add them to my recommended reading list for the patients I see.

David L. Dunner, M.D. University of Washington School of Medicine Seattle, Washington Center for Anxiety and Depression Mercer Island, Washington

\section{Psychiatric Disorders and Diabetes Mellitus edited by Maria D. Llorente and Julie E. Malphurs. Informa Healthcare, Abingdon, United Kingdom, 2007, 249 pages, $\$ 94.95$.}

Now that the links between major psychiatric illnesses and diabetes have been described through a substantial amount of original research, it's time for clinicians on both sides of the street, mental health specialists, and anyone who treats diabetes, to become familiar with this literature and its implications for treatment. This book examines the development, management, and prevention of diabetes mellitus in patients with psychiatric disorders.

Edited by 2 members of the Department of Psychiatry and Behavioral Science at the University of Miami, this collection of 13 chapters by 25 contributors (all but 2 from the University of Miami) covers a broad range of topics, including nutritional interventions, sexual dysfunctions, collaborative care, and psychosocial therapies. The first chapter reviews the metabolism and pharmacology of diabetes. Selecting two psychiatric illnesses for special attention, Chapter 2 focuses on diabetes and schizophrenia and Chapter 3 focuses on diabetes and depression.

This collection blends reviews of the research literature in the chapters on cognitive impairment, psychosocial treatments, and collaborative care with some clinically useful recommendations in the chapters on HIV, exercise, sexual dysfunction, and nutrition. The fascinating chapter on HIV infection, diabetes, and psychiatric disorders describes this underappreciated set of relationships and is bolstered by an ample reference list. In the chapter on psychopharmacology, the table on "psychotropic drug impact on diabetes" will be useful to psychiatrists and diabetologists.

The absence of an introduction identifying the problem and the audience to be addressed by this book leaves the reader guessing about the context for this collection. Though obesity is treated as a recurring theme in most chapters, the other missing piece is a chapter focusing on obesity and its relationship to psychiatric illness and diabetes. Aside from these minor shortcomings, this book provides a rich understanding of the complicated relationship between diabetes and psychiatric illness and will be helpful for any practitioner treating patients with these diseases.

Joseph M. Cerimele, B.A. Lawson R. Wulsin, M.D. University of Cincinnati College of Medicine Cincinnati, Ohio 